\title{
Criminologie
}

\section{Un nouvel enjeu de santé publique au Canada}

L'accompagnement des personnes endeuillées à la suite d'un suicide

\section{A new public health issue in Canada}

Helping people bereaved by suicide

\section{Una nueva apuesta de salud publica en Canadá El acompañamiento de las personas afligidas después de un suicidio}

\author{
Rae Spiwak, Brenda Elias, Jitender Sareen, Mariette Chartier et James M. \\ Bolton
}

Volume 51, numéro 2, automne 2018

Prise en charge du suicide : entre crime, troubles mentaux et droit de mourir

URI : https://id.erudit.org/iderudit/1054238ar

DOI : https://doi.org/10.7202/1054238ar

Aller au sommaire du numéro

Éditeur(s)

Les Presses de l’Université de Montréal

ISSN

0316-0041 (imprimé)

1492-1367 (numérique)

Découvrir la revue

Citer cet article

Spiwak, R., Elias, B., Sareen, J., Chartier, M. \& Bolton, J. M. (2018). Un nouvel enjeu de santé publique au Canada : l'accompagnement des personnes endeuillées à la suite d'un suicide. Criminologie, 51(2), 136-166. https://doi.org/10.7202/1054238ar

\section{Résumé de l'article}

Chaque année, dans le monde, entre 48 et 50 millions de personnes se retrouvent endeuillées à la suite d'un suicide. Au Canada seulement, ce sont 4000 individus qui se suicident chaque année, laissant derrière eux un grand nombre de personnes qui doivent faire face au caractère traumatisant et complexe du suicide. Dans un contexte qui avait déjà vu la tentative de suicide être décriminalisée en 1972, la présente étude se penche sur ce qui apparaît désormais constituer une nouvelle problématique de santé publique : le soutien aux personnes endeuillées à la suite d'un suicide. Nous discuterons à cet égard de quelques acteurs clés qui ont influencé les politiques canadiennes sur le sujet : les tribunaux, la Constitution ou encore des groupes qui avaient un intérêt spécifique par rapport à cet enjeu. Les questions de santé associées à ce type de deuil de même que la question de la nécessité de l'intervention seront abordées. Enfin, divers aspects émergents de ces politiques qui demandent encore à être éclairés seront examinés, de même que la nécessité de distinguer les approches à court, moyen et long terme quand on intervient auprès de personnes touchées par ce type de deuil. 


\title{
Un nouvel enjeu de santé publique au Canada
}

\section{L'accompagnement des personnes endeuillées à la suite d'un suicide}

\author{
Rae Spiwak, Ph. D. ${ }^{12}$ \\ Sciences de la santé communautaire, Université du Manitoba \\ rspiwak@gmail.com
}

Brenda Elias, Ph. D.

Professeure agrégée, Sciences de la santé communautaire, Université du Manitoba Brenda.Elias@umanitoba.ca

Jitender Sareen, M.D., FRCPC

Professeur, Psychiatrie, Psychologie et Sciences de la santé communautaire Université du Manitoba

Jitender.Sareen@umanitoba.ca

Mariette Chartier, R.N., Ph. D.

Professeure adjointe, Sciences de la santé communautaire, Université du Manitoba Chercheure scientifique principale, Centre d'élaboration de la politique des soins de santé du Manitoba Mariette_Chartier@cpe.umanitoba.ca

James M. Bolton, M.D., FRCPC

Professeur agrégé, Psychiatrie, Psychologie et Sciences de la santé communautaire Chercheur scientifique adjoint, Centre d'élaboration de la politique des soins de santé du Manitoba jbolton@exchange.hsc.mb.ca

\section{Traduit de l'anglais au français par Florence Dubois}

1. Department of Community Health Sciences, Université du Manitoba, 771, avenue Bannatyne, Winnipeg (Manitoba), Canada, R3E 3N4.

2. La rédaction de cet article a été soutenue par des subventions de recherche $\mathrm{du}$ Manitoba Health Research Council (Dr Bolton), une bourse du Manitoba Health Research Council Chair Award (Dr Sareen), une bourse CRSH Joseph-Armand Bombardier Canada et une bourse Manitoba Health Research Council Graduate Studentship (Mme Spiwak), ainsi qu'une bourse pour nouveau chercheur des Instituts de recherche en santé du Canada (Dr Bolton \# 113589). Les sources de financement n'ont joué aucun rôle dans l'élaboration et la conduite de cette étude: que ce soit dans la collecte, la gestion, l'analyse ou l'interprétation des données, mais aussi dans la préparation, la révision ou encore l'approbation de l'article. 
RÉSUMÉ - Chaque année, dans le monde, entre 48 et 50 millions de personnes se retrouvent endeuillées à la suite d'un suicide. Au Canada seulement, ce sont 4000 individus qui se suicident chaque année, laissant derrière eux un grand nombre de personnes qui doivent faire face au caractère traumatisant et complexe du suicide. Dans un contexte qui avait déjà vu la tentative de suicide être décriminalisée en 1972, la présente étude se penche sur ce qui apparaît désormais constituer une nouvelle problématique de santé publique: le soutien aux personnes endeuillées à la suite d'un suicide. Nous discuterons à cet égard de quelques acteurs clés qui ont influencé les politiques canadiennes sur le sujet: les tribunaux, la Constitution ou encore des groupes qui avaient un intérêt spécifique par rapport à cet enjeu. Les questions de santé associées à ce type de deuil de même que la question de la nécessité de l'intervention seront abordées. Enfin, divers aspects émergents de ces politiques qui demandent encore à être éclairés seront examinés, de même que la nécessité de distinguer les approches à court, moyen et long terme quand on intervient auprès de personnes touchées par ce type de deuil.

MOTS CLÉS • Suicide, politique, prévention, Canada.

\section{Introduction}

Tous les ans, dans le monde, entre 48 et 50 millions de personnes se retrouvent endeuillées à la suite d'un suicide (Pitman, Osborn, King et Erlangsen, 2014). Au Canada seulement, ce sont 4000 personnes qui se suicident chaque année (Association canadienne pour la prévention du suicide, 2017c), laissant derrière eux un grand nombre de personnes qui doivent faire face au caractère traumatisant et complexe du suicide (Bolton et al., 2013 ; Jordan et McIntosh, 2011a, 2011b; Sapsford, 1998; Sveen et Walby, 2008). On estime que le coût direct et indirect annuel du suicide au Canada dépasse 2,9 milliards de dollars (Agence de la santé publique du Canada, 2016). Le suicide représenterait 1,5\% du fardeau de la maladie à l'échelle mondiale, ou plus de 20 millions d'années de vies corrigées du facteur invalidité (Mann et al., 2005b). S'il est possible de calculer les coûts économiques du suicide, les répercussions sur les individus endeuillés par le suicide sont moins tangibles, bien que tout aussi importantes. Chaque suicide a en effet des conséquences profondes ressenties par la famille et l'ensemble des individus laissés dans le deuil. Mais alors que des études ont bien montré ces conséquences négatives et le besoin de services pour les individus endeuillés par le suicide (Bolton et al., 2013; Erlangsen et al., 2017; Jordan, 2008; Sveen et Walby, 2008), les politiques en santé publique sont restées, elles, très silencieuses sur ces enjeux et aucune politique nationale de prévention du suicide n'a d'ailleurs été mise en place. Au-delà de ce manque de stratégie criant, il faut néanmoins souligner l'apparition récente d'un cadre de travail fédéral pour la prévention du 
suicide. La présence d'un tel cadre suggère qu'une véritable politique canadienne en la matière est peut-être finalement enfin en cours (Gouvernement du Canada, 2016).

Les politiques en matière de suicide représentent en fait un domaine vaste qu'on peut scinder en trois approches: en amont, médianes et en aval. Les premières renvoient à des politiques générales de santé publique pour améliorer la santé de l'ensemble de la population (McKinlay, 1998). Les deuxièmes, de nature préventive, privilégient des interventions de type éducatif pour prévenir des maladies (McKinlay, 1998). Enfin, les approches en aval sont de nature curative et s'appliquent à des problèmes de santé après leur apparition (McKinlay, 1998). Parler de politiques en matière de suicide peut donc par exemple renvoyer à des études sur les facteurs de risque, à des interventions qui sensibilisent sur le suicide ou encore au soutien qui serait donné à des personnes qui ont tenté de mettre fin à leurs jours. Ces politiques peuvent aussi être sous-divisées en deux domaines: la prévention du suicide, qui a pour but de prévenir l'acte du suicide, et la postvention du suicide, qui a cette fois pour objectif de soutenir et d'assister les individus endeuillés par un suicide, notamment en essayant de limiter les répercussions néfastes d'un tel événement dans leur vie (Shneidman, 1981). Ces deux domaines se retrouvent donc dans les approches médianes et en aval décrites plus haut. Quoi qu'il en soit, toutes les composantes d'une politique sont importantes si on veut à la fois tenter de prévenir des suicides et accompagner des personnes endeuillées dans des situations d'après-suicide. De même, la connaissance de certains facteurs de risque peut aider à identifier des individus qui seront grandement affaiblis par le suicide d'un proche comme à repérer ceux qui pourraient bénéficier d'interventions particulières.

Le présent article parle de l'émergence d'un nouvel enjeu de santé publique au Canada: le soutien aux personnes endeuillées par le suicide d'un proche. Tout au long de notre réflexion, certains aspects des politiques visant les peuples autochtones seront discutés, même si ni le rôle important ni le point de vue des communautés autochtones dans la création des stratégies de prévention et de postvention du suicide ne seront évoqués en détail ${ }^{3}$. Concernant maintenant le présent article, nous survolerons d'abord quelques facteurs de risque entourant la

3. Des études comme celle de Connor, Rice et Leenaars (2017), qui s'appuie sur le regard des Premières Nations, constitueraient sans aucun doute une plus-value substantielle pour quiconque veut approfondir ces questions. 
question du suicide avant d'évoquer très brièvement ceux qui touchent les individus endeuillés par un passage à l'acte réussi. L'article montre ensuite comment certaines institutions politiques et juridiques ont conduit à façonner les politiques en matière de suicide telles qu'on les connaît actuellement, et avec quelles conséquences sur le plan plus général de la santé. Les particularités du système de santé canadien sont également évoquées dans le contexte de leurs conséquences sur le développement de politiques générales en santé et des progrès dans ce domaine. Avant de conclure, nous reviendrons sur les approches politiques en amont, médianes et en aval et discuterons plus spécifiquement de la présence d'idées innovantes en ce qui concerne les politiques à venir en matière de postvention du suicide.

\section{Quelques facteurs de risque entourant le suicide}

Les facteurs de risque du suicide sont multiples et interagissent de manière complexe. Ils comprennent une variété de facteurs de santé, sociaux et démographiques comme le sexe masculin et le genre (Fung et Chan, 2011; Hawton et van Heeringen, 2009; Qin, Agerbo et Mortensen, 2003), le jeune âge ou la vieillesse (Conwell, Duberstein et Caine, 2002; Conwell, Van Orden et Caine, 2011; Fung et Chan, 2011; Hawton et van Heeringen, 2009), le statut économique bas et le niveau d'éducation (Hawton et van Heeringen, 2009; Kessler, Borges et Walters, 1999), l'appartenance à une minorité ethnique (Bolton, Belik, Enns, Cox et Sareen, 2008; Katz et al., 2006; Kirmayer et al., 2007), l'isolement social (Conwell, Rotenberg et Caine, 1990; Durkheim, 1952; Van Orden et Conwell, 2011), le placement en "pensionnats autochtones» (Bombay, Matheson et Anisman, 2011) ou en foyers d'accueil (Katz et al., 2011), les violences familiales et à l'égard de mineurs (Devries et al., 2011; Gravseth, Mehlum, Bjerkedal et Kristensen, 2009; Mandelli, Carli, Roy, Serretti et Sarchiapone, 2011 ; McFarlane, Groff, O'Brien et Watson, 2003), les antécédents familiaux de troubles psychologiques incluant le suicide (Cheng, Chen, Chen et Jenkins, 2000; Gureje et al., 2010; Spiwak et al., 2011 ; von Borczyskowski, Lindbland, Vinnerlijung, Reintjes et Hjern, 2011), les troubles psychologiques comme tels (Bolton et al., 2008; Nock et al., 2009; Nock, Hwang, Sampson et Kessler, 2010; Sorensen et al., 2009), la douleur et la maladie physique (Conwell et al., 2011; Juurlink, Herrmann, Szalai, Kopp et Redelmeier, 2004; Leenaars et al., 1998; Waern, Rubenowitz et Runeson, 
2002), et bien d'autres facteurs (Hawton et van Heeringen, 2009; Kessler et al., 1999; Qin et al., 2003; Russell et Joyner, 2001). Si ces facteurs de risque peuvent contribuer au passage à l'acte suicidaire, une partie d'entre eux peut également affecter la santé des individus endeuillés. Comprendre lesquels de ces facteurs peuvent rendre ces individus vulnérables aidera à orienter les politiques générales à même d'identifier des individus à risque mais aussi à développer des approches qui cherchent à prévenir le suicide et ses conséquences sur plusieurs plans.

\section{Les politiques en matière de suicide au Canada}

Au cours des dernières décennies, le débat entourant la prévention du suicide a été assurément vif dans les politiques de santé à travers le monde. Au Canada, les politiques ont par ailleurs aussi touché à l'enjeu de la postvention. Prévention et postvention ont été articulées comme intrinsèquement liées et ont été mises pour cette raison sous le même cadre.

Jusqu'en 1972, la tentative de suicide était considérée comme un geste criminel (Leenaars et al., 1998). Avant cette date, le Code criminel de 1892 stipulait spécifiquement que «toute personne qui attente à sa propre vie est coupable d'un acte criminel passible de deux ans d'emprisonnement [traduction libre]» (Gouvernement du Canada, 1892). En 1954, la loi avait cependant réduit cette période d'incarcération à six mois (Penney, 2014). Ce n'est finalement qu'après sa complète décriminalisation que le suicide commence à être vu comme une problématique de santé. Et bien que cet acte sera rapidement classé dans ce nouveau registre de régulation, la sensibilisation politique sur la question continue à se faire attendre. Les individus endeuillés par le suicide sont néanmoins bientôt identifiés et surtout reconnus comme un groupe vulnérable sur le plan de la santé. Mais les programmes qui ont trait à la postvention du suicide restent sous-développés, voire non développés, et ce, en raison du caractère toujours largement embryonnaire du développement de politiques en matière de suicide au Canada (Spiwak, Elias, Bolton, Martens et Sareen, 2012).

Le développement de telles politiques au Canada en est en effet encore à ses balbutiements, comme l'indiquent entre autres un premier soutien explicite et non partisan de tous les partis politiques majeurs pour une stratégie nationale de prévention du suicide (Kirkup, 2011; Nation Talk, 2010) et la mise en place récente d'un cadre fédéral pour cette prévention (Gouvernement du Canada, 2016). Comme le définissent Howlett, 
Ramesh et Perl (2009) et Miljan (2008), les politiques en matière de santé se développent généralement en cinq étapes: la définition du problème que vise la politique, la formulation de celle-ci, le processus décisionnel, la mise en place de la politique et, enfin, son évaluation (Howlett et al., 2009; Miljan, 2008; Spiwak et al., 2012). Or, sur le suicide, le Canada n'a franchi que les deux premières étapes de ce cycle: 1) la clarification et la définition du problème; et 2) la formulation de la politique qui devra y répondre. À la suite de l'adoption récente du cadre fédéral travaillant à la prévention du suicide, on peut considérer que le pays entre maintenant dans sa troisième étape de développement des politiques, à savoir celle au cours de laquelle des décisions seront prises pour pouvoir ensuite mettre en action ces politiques dans la société (Spiwak et al., 2012). Si le Canada a longtemps eu du mal à franchir les autres étapes, c'est sans doute à cause du temps qu'a mis le suicide à émerger comme enjeu de santé publique (et donc aussi la question de l'accompagnement des personnes endeuillées après un passage à l'acte). Et si cette émergence a fini par se produire, c'est à la fois malgré et avec le concours de diverses institutions et structures politico-juridiques, incluant les tribunaux et la Constitution. Ces influences ont en effet mené à l'absence d'une stratégie fédérale de prévention du suicide, mais également à des carences criantes en termes de directives et de réglementation en ce qui a trait au soutien et au suivi des personnes endeuillées à la suite d'un suicide.

\section{Les institutions}

Les institutions vont à la fois faciliter et contraindre la mise au point de politiques en matière de suicide (Howlett et al., 2009), notamment concernant l'accompagnement des personnes endeuillées. Ces institutions, en particulier les tribunaux mais aussi la Constitution, ont chacune joué un rôle important dans la manière dont nous voyons aujourd'hui le suicide (comme la façon d'y répondre), et ce, sur les différents plans mentionnés plus haut.

\section{Les tribunaux}

Quand le suicide était encore considéré comme un crime, les politiques qui l'abordaient étaient regroupées sous les instances fédérales. Mais à la suite de la décriminalisation de sa tentative en 1972, le suicide va de plus en plus être vu comme un problème de santé. Certains décideurs 
politiques vont alors exercer des pressions sur le gouvernement pour que des politiques de prévention et de promotion de la santé soient désormais mises en place (Lalonde, 1974).

Le suicide ne relevant plus de la compétence fédérale, les programmes et services qui y sont liés se trouvent désormais placés sous la responsabilité des provinces. Or les programmes de prévention et de postvention du suicide sont souvent bien loin d'être adéquatement répartis entre les provinces, régions et territoires. Si l'on peut souligner qu'il en va de même pour presque tous les services de santé au Canada, la nature sociale du suicide et sa stigmatisation aggravent les disparités, créant davantage de difficultés pour les individus qui cherchent à accéder à des services organisés. De plus, si le suicide est décriminalisé, il demeure encore socialement stigmatisé par son ancienne connotation criminelle, comme le montre d'ailleurs la formulation populaire «commettre un suicide». Cette phrase reste en effet toujours aussi utilisée aujourd'hui alors même qu'elle sousentend qu'une personne qui a mis fin à sa vie a de ce fait commis un crime. Garder une telle formulation ne peut dès lors qu'entretenir l'image du suicide comme celle d'un acte odieux appelant un jugement lourdement négatif, ce qui ne va pas sans aggraver les conséquences de la stigmatisation qui y est déjà liée (Sommer-Rotenberg, 1998).

Quoi qu'il en soit, il apparait en tout cas clair que les programmes de prévention finalement mis en place ont été influencés par les tribunaux et en particulier par l'interprétation juridique du suicide. Avant la décriminalisation de la tentative de suicide en 1972 (et celle du suicide en 1892), cet acte était conçu comme illégal et punissable par la loi. Les perceptions individuelles de l'acceptabilité d'un comportement suicidaire sont donc longtemps restées imprégnées de ces conceptions, tout comme les décisions des gouvernements qui devaient choisir comment orienter les ressources et leur budget. Si accorder des ressources à des services répondant à un acte illégal était sans doute difficile à défendre sur le plan gouvernemental, avoir le soutien du public devait l'être davantage encore quand on sait qu'à l'opprobre pénal s'ajoutait la stigmatisation du suicide par l'Église (Public Initiative to Prevent Suicide and Self-Harm, 2007).

\section{Le gouvernement fédéral et l'Acte constitutionnel}

Le gouvernement fédéral et l'Acte constitutionnel sont des structures institutionnelles importantes qui ont guidé la responsabilité de gérer et de fournir des services de santé. Ces institutions ont aussi influencé la 
manière dont les politiques sur le deuil à la suite d'un suicide ont émergé comme une question de santé publique. Deux ans après la décriminalisation de la tentative de suicide au Canada, l'honorable Marc Lalonde définit ainsi le suicide comme un important enjeu de santé publique qui mérite de l'attention (Lalonde, 1974). L'inclusion dans son rapport de politiques concernant le suicide aura été un tournant préparant le terrain pour d'autres avancées de cette problématique au Canada. Si le rapport Lalonde n'a pas mis d'accent spécifique sur le besoin de soutien pour les individus endeuillés par le suicide, il a du moins reconnu les sentiments que peuvent avoir ces derniers, témoignant ainsi d'une reconnaissance de la part du gouvernement (Lalonde, 1974).

Plusieurs années après le rapport, le gouvernement fédéral répond par l'entremise de Santé et Bien-être social Canada qui établit le groupe d'étude national sur le suicide au Canada. En 1987, ce groupe génère son premier rapport, intitulé Le suicide au Canada: Rapport du groupe d'étude national sur le suicide au Canada (Santé Canada, 1987).

Ce rapport national soulignait la question des endeuillés après un suicide, mais aussi de la postvention en insistant sur l'importance à la fois de politiques à l'échelle systémique et d'un réseau intégré de soins sur les plans fédéral, provincial, territorial et régional. Plusieurs années plus tard, une commission royale se penche sur le suicide au sein des peuples autochtones du Canada (Parlement du Canada, 1995). Comme la santé des Autochtones est sous le mandat du fédéral, cette démarche ne fait pas face aux mêmes problèmes de juridiction que les programmes de santé mandatés au provincial. Cette commission mène à la création de stratégies de prévention visant la santé des Autochtones, stratégies qui incluent la création de la Stratégie nationale de prévention du suicide chez les jeunes Autochtones (Santé Canada, 2003). Centrée autour de la prévention, cette stratégie va néanmoins également inclure la postvention et le soutien aux personnes endeuillées, chaque fois en cherchant à tenir compte de facteurs culturels propres aux Premières Nations. Bien que la création de cette stratégie en 2005 constitue sans nul doute une avancée importante en ce qui a trait aux politiques générales sur le suicide, elle attire aussi l'attention sur le fait qu'à la même époque, il y a un manque criant de programmes de prévention et de postvention du suicide pour des populations non autochtones. En 2006, le gouvernement fédéral dépose un document clé, le rapport Kirby, qui met en lumière les problématiques liées à la santé mentale et la question de leur régulation par les juridictions fédérales ou provin- 
ciales (Sénat du Canada, 2006). Ce rapport reconnaît ainsi que le suicide est un thème prioritaire en santé et recommande la création d'une stratégie nationale de prévention du suicide qui appellerait à la collaboration fédérale, provinciale et territoriale avec d'autres instances. La recommandation d'un service pour les individus endeuillés par le suicide et l'idée d'inclure cette question dans la stratégie de prévention à l'échelle nationale sont particulièrement mises en avant.

Dans un contexte qui conduit le gouvernement à devoir faire face à la question de la santé mentale au Canada tout en respectant les ordres de compétence juridictionnelle inhérents au gouvernement du pays, la Commission de la santé mentale du Canada (CSMC) est créée, répondant ainsi aux recommandations du rapport Kirby de 2006 (Sénat du Canada, 2006). Conçue pour permettre au gouvernement d'opérer à distance, la CSMC se compose d'employés non gouvernementaux (Sénat du Canada, 2006). Bien qu'elle ait été pensée pour compléter les structures gouvernementales fédérales, provinciales et territoriales (Sénat du Canada, 2006), elle demeure un programme national qui a besoin d'être adopté par ces trois ordres pour être mis en pratique. Un des principaux buts de la CSMC consiste à réduire la stigmatisation sociale associée à la maladie mentale. Sa stratégie en matière de santé mentale pour le Canada, Changer les orientations, Changer des vies, comprend plusieurs recommandations visant à faire significativement progresser la prévention du suicide, notamment en ce qui concerne le soutien aux familles endeuillées (CSMC, 2012). Voir le suicide inclus dans cette politique nationale en matière de santé mentale constitue indéniablement un pas considérable vers un programme réglementé de prévention et de postvention du suicide, soit un programme qui aura été longtemps négligé par les législations passées.

Une autre grande avancée dans l'esquisse d'une politique canadienne sur le suicide implique que le gouvernement fédéral détermine des priorités de recherche dans ce domaine. En 2003, le gouvernement s'est ainsi concentré sur la sensibilisation et a fait du financement de telles recherches une priorité. Santé Canada et l'Institut des neurosciences, de la santé mentale et des toxicomanies des Instituts de recherche en santé du Canada (IRSC) ont tenu une conférence pour souligner l'importance de la recherche sur le suicide (Instituts de recherche en santé du Canada, 2003). Rappelant le lien entre santé mentale et suicide, cette conférence a réitéré l'importance d'une approche fondée sur des données probantes en vue de promouvoir la première et de prévenir 
le second (Instituts de recherche en santé du Canada, 2003). Les activités précédentes liées à cette question au Canada jumelées à un appel à la recherche et à un soutien de celle-ci montrent combien le gouvernement reconnaît désormais le suicide comme un enjeu incontournable de santé publique au pays. Plus récemment, le cadre fédéral de prévention du suicide s'est concentré sur la recherche comme un moyen d'augmenter la prise de conscience et de réduire la stigmatisation sociale (Gouvernement du Canada, 2016). Même la Défense nationale et les Anciens Combattants du Canada (ACC) ont mis en place une stratégie de prévention du suicide pour les Forces armées canadiennes et la communauté des vétérans (Gouvernement du Canada, 2017). Cette stratégie met l'accent sur la prévention du suicide comme un enjeu de santé publique prioritaire pour ces populations à risque.

C'est donc grâce aux gouvernements fédéral et provinciaux que s'installe bientôt une politique générale sur le suicide à travers tout le pays; une politique qui ouvre à son tour diverses voies concernant la prévention. À cet égard, si plusieurs approches médianes et en aval existent en matière de prévention et de postvention du suicide, les approches en amont sont nettement plus rares. Pour ce qui a trait aux approches médianes, un appel national à la recherche sur le suicide (Instituts de recherche en santé du Canada, 2003) accompagne la création de stratégies de promotion de la santé (Instituts de recherche en santé du Canada, 2006). Ces approches, comme celles en aval, incluront également des campagnes de déstigmatisation de la santé mentale, notamment par une formation spécialisée donnée aux professionnels de la santé et une attention particulière accordée à la prévention du suicide dans l'armée et pour les populations des Premières Nations (Commission de la santé mentale du Canada, 2011; Gouvernement du Canada, 2017; Santé Canada, 2003). En somme, le gouvernement aura joué un rôle notable dans la création de politiques de prévention du suicide, peut-être à même d'encourager un changement positif dans la perception individuelle et sociale du suicide.

\section{Les groupes d'intérêt spécifiques}

Bien que le gouvernement ait été actif dans le domaine des politiques en matière de suicide, que ce soit en formulant des stratégies nationales pour prévenir ce phénomène chez les Autochtones, en mettant sur 
pied la CSMC, en exigeant des rapports nationaux en la matière ou encore en posant le cadre fédéral de prévention du suicide, peu d'attention a été accordée aux besoins de ceux qui se retrouvent endeuillés à la suite d'un suicide. En plus d'être attribuable aux tribunaux et à la Constitution, le retard dans cet effort s'explique peutêtre également par un manque de reconnaissance de la part des fournisseurs de soins de santé (ainsi que par le manque de programmes de traitement qui en découle) (Nishimaki et al., 2009). S'ajoutent à cela la compréhension limitée du suicide et son effet stigmatisant (McMenamy, Jordan et Mitchell, 2008) pour la majorité des gens. Si le gouvernement a tardé à réagir pour accompagner ces personnes endeuillées, les expériences de ces dernières n'en ont pas moins servi de guide pour aider à la création de groupes de soutien au début des années 1980 au Canada. Le premier groupe de la sorte est formé par le Clarke Institute of Psychiatry et des centres d'aide et d'écoute de Toronto, le tout sous la direction de Karen Letofsky en 1979 (Edmunds, 1998; Rosenfeld, 1998). Après la décriminalisation de la tentative de suicide se développe une sensibilité accrue à la postvention, qui se traduit par la mise en place de groupes de soutien mais aussi par l'émergence de questions propres au caractère unique du deuil à la suite d'un suicide (Ambrose, 1998; Rosenfeld, 1998; Sapsford, 1998). Et alors que le gouvernement continue à délaisser ces questions, des groupes d'intérêt nationaux comme l'Association des psychiatres du Canada (APC) et l'Association canadienne pour la prévention du suicide (ACPS) joueront un rôle actif dans la sensibilisation à ce phénomène, que ce soit dans le domaine de la prévention ou dans celui du deuil.

L'APC a poussé les revendications jusqu'à créer des directives médiatiques pour le signalement des suicides dans le but de réduire la contagion et la stigmatisation du geste (Nepon, Belik, Bolton et Sareen, 2010). L'ACPS a été créée en 1985 par des professionnels qui reconnaissent la nécessité de réduire le taux de suicide et de fournir de l'aide aux individus endeuillés. Elle joue un rôle important dans la promotion de politiques à mettre en œuvre par tous les ordres de gouvernement, tout comme dans le partage et l'accroissement des connaissances en intervention et en recherche sur le suicide (Association canadienne pour la prévention du suicide, 2017a). En 2004, le président de l'Association en appelle au leadership fédéral pour que soit développée une stratégie nationale de prévention du suicide, soutenant que tous les ordres gouvernementaux devraient s'impliquer s'ils espèrent réduire le taux 
de suicide au Canada (Sénat du Canada, 2005). Récemment, la progression vers une telle stratégie s'est faite plus activement, avec le soutien impartial de tous les partis politiques majeurs (Nation Talk, 2010). Le cadre fédéral de prévention du suicide qui en a résulté stipule clairement que les individus endeuillés par le suicide devraient être davantage impliqués dans le développement des directives et des ressources (Gouvernement du Canada, 2016). Ce cadre suggère par ailleurs que ces directives et ces ressources devraient être prioritaires dans la recherche en matière de prévention du suicide. L'ensemble de ces développements récents constituent un progrès indéniable et témoignent de l'engagement du gouvernement sur plusieurs plans. Celui-ci prend enfin conscience du problème du suicide (incluant la question du deuil) et des barrières structurelles qui restent à surmonter pour le Canada.

Des groupes d'intérêt spécifiques se sont impliqués dans diverses activités en matière de prévention du suicide qui rassemblent des approches en amont, médianes et en aval. Ils ont eu une influence sur d'autres institutions, donnant une voix à la sensibilisation sur le sujet et aidant à combattre la stigmatisation sociale avec l'aide et l'expertise de professionnels. Ce soutien et ces connaissances sont loin d'être négligeables quand il est question d'influencer les politiques gouvernementales et la vision de la société en matière de prévention du suicide. Les groupes en question jouent ainsi un rôle prépondérant dans ces politiques tout en pouvant faciliter les initiatives de soutien envers les individus endeuillés. Ces groupes participent à la légitimation de ces initiatives et peuvent diriger les individus vers les services compétents.

\section{Nouvelles politiques en matière de suicide qui demandent encore à être éclairées}

Le rôle d'une stratégie nationale en matière de prévention du suicide et la systématisation des programmes de postvention

En 1993 a lieu à Calgary, en Alberta, une rencontre internationale sur la prévention du suicide, rencontre tenue par les Nations Unies durant laquelle des directives sont établies pour créer une stratégie nationale de prévention du suicide (Agence de la santé publique du Canada, 2005). Si de nombreux pays ont suivi ces directives pour créer de telles stratégies, le Canada est resté le seul à ne pas s'être encore engagé dans ces démarches, peut-être politiquement bloqué en matière de santé à 
cause du problème multi-juridictionnel de la division des responsabilités provinciales et fédérales. Une stratégie nationale en matière de prévention du suicide permettrait pourtant aux deux ordres gouvernementaux de travailler de concert, tant sur la prévention que sur la postvention. Plusieurs recommandations avancées par le Rapport du groupe d'étude national sur le suicide au Canada pourraient ainsi être mises en œuvre (Santé Canada, 1987).

La concrétisation d'une stratégie nationale de prévention du suicide (incluant les enjeux du deuil) relève néanmoins d'une tâche immense, à commencer par son intégration à des structures provinciales souvent redondantes en matière de prévention du suicide, mais aussi par le fait qu'elle demanderait de créer des directives et des règles uniformes pour les initiatives de soutien. Par exemple, au Manitoba, plusieurs structures de prévention du suicide existent, mais chacune répond à des questions similaires (Martens et al., 2008). Il existe aussi plusieurs groupes de soutien pour des personnes endeuillées par un suicide mais qui ne sont régulés et organisés ni par le gouvernement ni par des groupes professionnels (Association canadienne pour la prévention du suicide, 2017b). L'Organisation mondiale de la santé (OMS) a pourtant émis des directives sur la manière de créer de tels groupes de soutien, mais celles-ci ne sont pas suivies et reposent sur une base volontaire, ce qui pousse à remettre en question leur qualité. Ces directives reconnaissent l'importance, dans l'ensemble du processus, du rôle de professionnels et de spécialistes de l'éducation pouvant former des membres à mieux comprendre le deuil ou d'autres éléments essentiels à la tenue d'un groupe (OMS, 2008). Les directives abordent également la création d'un organisme-cadre qui superviserait les groupes afin d'encourager leur pérennité (OMS, 2008). Si l'OMS propose ces directives, elles ne sont pas officiellement réglementées. Des services de soutien peuvent donc être créés et mis en place par des individus endeuillés par le suicide; or, certains peuvent ne pas être émotionnellement outillés ou assez formés pour diriger de tels groupes. Les plus récents développements du cadre fédéral de prévention du suicide indiquent que les directives de l'OMS ont inspiré sa structure actuelle, mais stipulent clairement qu'il ne s'agit pas d'une stratégie nationale et qu'elle ne remplace pas non plus les structures et stratégies mandatées au provincial ou ailleurs (Gouvernement du Canada, 2016).

Bien que les problèmes juridictionnels de mise en pratique d'une stratégie nationale de prévention du suicide soient inévitables compte tenu de l'Acte constitutionnel, le Canada doit peut-être s'inspirer des 
stratégies d'un autre pays pour trouver d'éventuelles solutions. Par exemple, les structures nationales établies en Australie pour la prévention du suicide sont mises en pratique par le fédéral, mais les gouvernements des États et des Territoires ont aussi leurs responsabilités déterminées par le Conseil des gouvernements australiens (Australian Government, 2008). Ce Conseil est un organisme qui rassemble le gouvernement fédéral, les gouvernements des six États et des deux Territoires principaux, ainsi que l'Association des gouvernements locaux (Australian Government, 2008). Il se rapproche de la Conférence des premiers ministres au Canada. Le premier ministre australien préside donc cet organisme dont le but est de discuter des activités gouvernementales à chacun de ses ordres et de les coordonner. En Australie, le gouvernement a brillamment réussi à ramener la question du deuil au cœur de la stratégie nationale de prévention du suicide. Il a établi des repères et émis des directives pour les groupes de soutien. Ces directives aident non seulement les organisateurs de tels groupes, mais soutiennent aussi leurs membres en incorporant les exigences législatives et de gestion applicables aux groupes de soutien (Australian Government, 2009). Les groupes en question sont formés en fonction de modèles clairement définis qui s'adaptent à la culture et favorisent le ressourcement (Australian Government, 2009). En plus des directives, des démarches alternatives sont proposées aux groupes qui peuvent se servir du téléphone ou encore d'Internet dans leur modèle. Toutes ces directives seraient pertinentes et adaptables à une stratégie nationale canadienne de prévention du suicide. Même si ces modèles se fondent sur des approches médianes ou en aval, la santé des individus qui ont besoin de soutien étant déjà à risque, ils n'en permettraient pas moins de régulariser les approches entre provinces et régions, ce qui entraînerait des normes de soins équivalentes pour tous les Canadiens. Un tel plan permettrait de débattre de l'intégration des ressources, de la réduction des doubles emplois et de l'amélioration de l'efficacité.

Outre l'Australie, d'autres pays ont adopté des approches fonctionnant sur plusieurs ordres gouvernementaux dans leurs stratégies nationales de prévention du suicide. Ces approches en appellent à la participation des institutions, agences et organismes nationaux et régionaux de la santé publique tels que les lignes de crise et les associations spécialisées en santé mentale (OMS, 2002). Des pays tels que la Finlande et la Norvège ont eux aussi utilisé ces approches et ont même impliqué les universités, les hôpitaux, la police et le système 
scolaire (OMS, 2002). Il est en tout cas clair que les pays dépourvus de stratégies nationales de prévention devraient utiliser des méthodes variées dans leurs approches régionales en matière de prévention du suicide, comme cela a été le cas au Canada, dans lequel il existe plusieurs cadres de prévention du suicide (Tanney, 1995).

Plusieurs États ont vu leurs efforts couronnés de succès dans l'élaboration de stratégies de prévention du suicide tout en connaissant des climats politiques divers. Les différences politiques entre les pays qui ont mis sur pied une stratégie nationale sautent plutôt aux yeux, comme le prouve la question de la juridiction provinciale et fédérale sur les questions de la santé pour le Canada. Malgré ces différences entre pays, des similarités émergent sur le plan des démarches de chacun pour le développement et la mise en pratique desdites stratégies. On pourrait penser que l'Acte constitutionnel du Canada fait preuve d'une complexité absente dans les autres pays. Mais même si les Canadiens tiraient avantage d'une approche gouvernementale sur plusieurs plans, elle serait sans doute bien difficile à mettre en pratique. Néanmoins, il demeure important de chercher à s'inspirer d'autres pays pour des exemples d'application de politiques et de programmes de prévention et de postvention du suicide dans des environnements multijuridictionnels. Les approches futures devraient non seulement tenir compte de la configuration politique d'un pays comme le Canada, mais aussi rendre justice à la diversité culturelle. Comme exemple de contribution importante aux politiques et programmes en question, notons l'élaboration de programmes de prévention du suicide chez les Autochtones qui intègrent la vision du monde, la perspective et les pratiques de guérison des Premières Nations (Connors et al., 2017). La Swampy Cree Suicide Prevention Team du Manitoba est un autre exemple de partenariat communautaire. Ce partenariat entre une université et des Premières Nations s'intéresse au développement de stratégies de prévention du suicide dans les communautés de ces dernières (Swampy Cree Suicide Prevention Team, 2008).

\section{Le rôle de la CSMC dans les politiques sur le suicide}

La stratégie nationale de prévention du suicide chez les populations des Premières Nations constitue un excellent exemple de l'utilisation d'approches en amont, médianes et en aval dans la prévention et la postvention du suicide. Parmi ses recommandations, cette stratégie 
comprend l'implication du gouvernement sur tous les plans de la prévention, incluant la promotion de la santé mentale, la réduction des risques à travers l'éducation publique, la réduction de l'accès à des moyens létaux au sein des communautés, une supervision et des conseils en période de crise aux individus qui expriment des pensées suicidaires, ou encore un soutien aux personnes endeuillées à la suite d'un suicide (Santé Canada, 2003). Si les politiques pour les individus non autochtones ne peuvent être abandonnées sur le plan fédéral, il se peut que d'autres groupes puissent diriger et superviser une telle stratégie sans compromettre la Constitution. En Australie, les populations autochtones partagent des facteurs de risque accrus similaires à ceux des Premières Nations du Canada. Si l'Aboriginal Health Division (Division de santé autochtone) du gouvernement australien a créé une stratégie adressée aux jeunes en 1998 (Youth Suicide Advisory Committee, 1998), le gouvernement cherche à présent à répondre aux besoins des communautés autochtones endeuillées avec l'intention de créer un système de soutien culturellement adéquat pour ces individus (Hawton, 2005). Bien que l'intégration de services fédéraux et provinciaux en santé continue à poser des difficultés au Canada, la CSMC pourrait bien réussir à jouer un rôle clé dans la création et la mise en place d'une stratégie de prévention du suicide, qu'elle tienne du couplage suicide/ santé mentale ou encore de la nature à la fois nationale et indépendante de l'organisme.

\section{Les obstacles à la postvention}

Qu'en est-il des conséquences en matière de santé d'un deuil après suicide si on le compare à d'autres formes de deuil? Des travaux ont montré des différences entre groupes endeuillés en examinant les taux respectifs de troubles mentaux et de comportements suicidaires. Des études canadiennes (Bolton et al., 2013, 2016), danoises (Agerbo, 2005 ; Erlangsen et al., 2017; Kessing, Agerbo et Mortensen, 2003 ; Qin et Mortenson, 2003) et suédoises (Kuramoto et al., 2010; Kuramoto, Brent et Wilcox, 2009; Omerov, Steineck, Nyberg, Runeson et Nyberg, 2013; Tidemalm et al., 2011) ont observé des populations entières et ont trouvé des différences psychiatriques selon le type de deuil, et ce, après avoir neutralisé des facteurs de pré-deuil tels que des troubles mentaux préexistants. Si ces recherches indiquent que les personnes endeuillées par le suicide sont plus à risque de voir 
leur santé en souffrir, celles-ci ne reçoivent pas nécessairement les services de postvention ou de soins qu'il leur faut en raison de divers obstacles. Ces obstacles peuvent inclure des éléments attitudinaux comme le fait de voir une personne endeuillée craindre ou vouloir éviter les services de santé mentale en raison de son embarras ou d'une stigmatisation redoutée, mais aussi tout simplement renvoyer au constat d'un manque de programmes de postvention efficaces (Sareen et al., 2007).

\section{La stigmatisation}

En vue de combattre la stigmatisation qui entoure encore la maladie mentale, la CSMC lance en 2009 Changer les mentalités, un programme de lutte contre la stigmatisation étalé sur dix ans. Le but de l'initiative: améliorer le sort des personnes souffrant de troubles mentaux et accroître le nombre d'entre elles qui vont chercher de l'aide (CSMC, 2011). Un des buts de Changer les mentalités consiste d'abord à cibler les fournisseurs de soins de santé puisque des études montrent que ceux-ci partagent les préjugés du reste de la population, y compris lorsqu'ils souffrent eux-mêmes d'une dépression (Adams, Lee, Pritchard et White, 2010). Pour les personnes endeuillées s'ajoute donc à la vulnérabilité de devoir faire face à un suicide la stigmatisation sociale toujours associée à celui-ci, avec pour conséquence un plus grand risque de vivre des dépressions majeures (Brent, Melhem, Donohoe et Walker, 2009; McMenamy et al., 2008; Schaal, Elbert et Neuner, 2009; Spiwak et al., 2011). Ces personnes apparaissent dès lors réticentes à aller vers les ressources en postvention, ce qui explique la nécessité d'inclure des approches en amont, médianes et en aval. Privilégier une approche populationnelle pour faire face à des questions de santé mentale à grande échelle ne constitue pas en soi une nouvelle approche. En 1974, le rapport Lalonde mettait déjà l'accent sur l'obligation d'aller au-delà d'un système de santé axé sur la maladie si l'on souhaitait améliorer la santé du public (Lalonde, 1974). À l'aide d'approches populationnelles, comme le programme Changer les mentalités, et d'approches sur le plan individuel qui ciblent, elles, des groupes spécifiques, il serait sans doute possible d'améliorer significativement la santé des personnes endeuillées par le suicide. 


\section{Efficacité de la postvention}

De manière générale, les interventions en matière de deuil à la suite d'un suicide ont été peu étudiées (Cerel, Padgett, Conwell et Reed, 2009; Jordan, 2015 ; Jordan et McMenamy, 2004). Les rares études faites évoquent un soutien mixte de prévention et de postvention du suicide, rendant difficile la détermination d'approches préventives qui fonctionnent bien (Mann et al., 2005a; OMS, 2004) tout comme celle d'interventions efficaces en postvention (Jordan, 2015; Jordan et al., 2011 ; McDaid, Trowman, Golder, Hawton et Sowden, 2008; Szumilas et Kutcher, 2011). En ce qui concerne cette dernière, deux synthèses systématiques reconnaissent une certaine pertinence des interventions touchant le deuil à la suite d'un suicide, mais les recherches sur la question tendent à être de qualité moindre; elles sont très descriptives et donc sans réelle dimension analytique (Jordan, 2015 ; McDaid et al., 2008; Szumilas et Kutcher, 2011). Les programmes de postvention du suicide incluant des plans d'évaluation sont par ailleurs rares et souvent mal conçus sur le plan structurel, méthodologique et statistique (Szumilas et Kutcher, 2011). Certaines études ont néanmoins montré que des individus endeuillés après un suicide qui avaient bénéficié de ces interventions ont vu leur anxiété et leur dépression reculer en plus de connaître une amélioration générale de leur expérience du deuil (Farberow, Gallagher-Thompson, Gilewski et Thompson, 1992; Jordan et al., 2011; Kovac et Range, 2000; Pfeffer, Jiang, Kakuma, Hwang et Metsch, 2002). Si des preuves de la pertinence des programmes de postvention ne sont pas légion, des études indiquent en tout cas qu'ils peuvent être bénéfiques (Cerel et Campbell, 2008; Constantino et al., 2001; Farberow et al., 1992; Jordan, 2015). Il faut bien sûr mener d'autres travaux pour déterminer si ce type d'interventions est réellement efficace et généralisable à des groupes ethniques variés tels que les Premières Nations (Jordan et McMenamy, 2004; McDaid et al., 2008). Cela peut sembler constituer un bien grand défi, mais les recherches et les évaluations dans le domaine pourraient fournir aux décideurs politiques les preuves dont ils ont besoin pour soutenir les initiatives de postvention du suicide, et ce, malgré les contraintes fiscales actuelles. Une étude de l'efficacité des traitements qui comprend une analyse des coûts/bénéfices serait d'ailleurs très utile aux décideurs, comme le seraient des études des changements notés dans la santé à la suite de la mise en place d'une stratégie nationale de prévention du suicide. 


\section{Des approches innovantes en postvention du suicide}

Afin de surmonter les obstacles susmentionnés et pouvoir répondre aux besoins des individus endeuillés par le suicide tout en établissant des stratégies innovantes qui préviennent et traitent les problèmes de santé, les politiques canadiennes à venir devraient découler d'une approche à plusieurs facettes. Elles doivent intervenir de manière équilibrée en amont, sur le plan médian et en aval (Gouvernement du Canada, 2016). Les individus endeuillés peuvent être par exemple ciblés par des approches en aval dont le but serait de répondre aux effets propres à ce type de deuil. De tels efforts incluraient la postvention, le soutien des pairs, des services de suivi et des groupes de soutien et de renseignement en ligne (Gouvernement du Canada, 2016). Inversement, il est également possible de mettre en œuvre des approches médianes axées sur des politiques de santé publique, notamment la promotion de la santé mentale (par exemple, en donnant de l'information aux jeunes au cours de leurs études postsecondaires, ce qui, cela serait prouvé, réduirait les suicides [Hawton et van Heeringen, 2009; Kessler et al., 1999]). Quoi qu'il en soit, les approches médianes constitueraient elles aussi une opportunité idéale pour des innovations variées, et ce, en vue de prévenir le déclin de la santé de personnes endeuillées à la suite d'un suicide. Il existe par exemple aux États-Unis une journée nationale pour ce type de deuil. Cette journée sensibilise la population à cette cause tout en favorisant toute initiative de réseautage. Une telle approche s'inscrit donc à la fois en aval et sur le plan médian puisqu'elle soutient les endeuillés tout en cherchant à directement renforcer le soutien de la collectivité à leur égard.

Les approches en amont, médianes et en aval

Campagnes de sensibilisation et incitatifs à la santé

En visant la promotion de la santé avec pour cible l'ensemble de la population, les approches en amont peuvent indéniablement avoir une influence vaste sur la santé populationnelle (McKinlay, 1998; Rose, 1985). Tout en touchant un public large, ces approches ont la capacité d'apporter un soutien individuel qui peut encourager les comportements sains. Le gouvernement peut notamment aider financièrement des personnes endeuillées à commencer ou à poursuivre des études supérieures. Par exemple, des personnes ayant fait leurs études secondaires 
ou postsecondaires seraient admissibles à des crédits d'impôt pour études ou à d'autres avantages. Ces incitatifs encourageraient ces individus à rechercher un niveau d'éducation plus élevé, ce qui améliorerait leur situation socioéconomique et réduirait le risque de voir leur santé souffrir. De même, le gouvernement pourrait emboîter le pas à la Suède et choisir d'ajouter d'autres incitatifs comme rendre gratuites les études postsecondaires (Indo European, 2011).

Mais des approches médianes générales, comme l'éducation sur la question du suicide et de la santé mentale ou encore les campagnes de sensibilisation, constitueraient elles aussi un moyen efficace de communiquer avec les Canadiens sur ces sujets délicats, notamment sur la stigmatisation trop souvent associée à la maladie mentale. Plusieurs études ont bien montré à cet égard comment l'élimination des troubles mentaux pouvait contribuer à réduire le taux de suicide (Beautrais, 2002; Yin Bun, Law, Chan, Liu et Yip, 2006). Même si les objectifs de promotion de la santé mentale et de sensibilisation à la question du suicide peuvent être en grande partie atteints grâce à ces approches, des outils doivent être créés pour accompagner le gouvernement dans l'organisation de campagnes et de programmes efficaces. Par exemple, l'approche de la prestation directe adoptée par les gouvernements fédéral ou provinciaux pourrait servir à engager des spécialistes de la santé publique et de la santé mentale pour organiser des campagnes qui sensibilisent efficacement à la fois sur le bien-être mental et sur le suicide. Ces campagnes pourraient cibler la population générale et des groupes plus spécifiques, comme les individus souffrant de troubles mentaux ou les individus qui ont perdu un proche par suicide. Pour ces campagnes éducatives, on peut penser ici autant à des approches médianes qu'à des interventions en aval. Les campagnes incluraient les populations à risque comme les communautés rurales ou nordiques, qui ont sans doute besoin d'interventions et de programmes différents de ceux mis en place pour les communautés urbaines. De tels efforts sont déjà inclus dans le cadre fédéral le plus récent (Gouvernement du Canada, 2016).

\section{La postvention active}

Une composante importante des approches médianes et en aval a trait aux modèles actifs de postvention. Dans la mesure où les personnes endeuillées à la suite d'un suicide ne cherchent généralement pas de soutien, la postvention active implique qu'il faut dès lors tenter de les 
contacter directement, que ce soit à des fins de prévention ou même d'intervention (Jordan et al., 2011). Une option consiste par exemple à les contacter juste après que le suicide a eu lieu. Dans un tel cas de figure, le bureau du médecin légiste identifierait les individus en deuil et serait responsable de leur proposer un service de suivi. Ces services pourraient inclure une évaluation de la situation de deuil durant laquelle les personnes seraient vues par un professionnel de la santé mentale et suivies ensuite pour s'assurer que les soins nécessaires leur sont prodigués. Un programme de ce type, le LOSS (Cerel, McIntosh, Neimeyer, Maple et Marshall, 2014; Jordan, 2015) existe déjà et a été mis sur pied aux États-Unis. Y sont offerts, au moment où le suicide est découvert, le soutien d'un professionnel en santé mentale et celui d'un bénévole qui s'est lui-même retrouvé endeuillé à la suite d'un suicide. Ces modèles actifs de postvention sont en tout cas de plus en plus reconnus (Campbell, Cataldie, McIntosh et Millet, 2004; Cerel et Campbell, 2008; Jordan et al., 2011) pour l'apport qu'ils offrent à des personnes endeuillées traumatisées qui peuvent difficilement aller chercher ellesmêmes de l'aide par manque de motivation (Jordan et al., 2011). Dans un autre registre certes, il a par ailleurs déjà été montré qu'une stratégie visant à recourir à des visites à domicile postnatales pouvait s'avérer payante pour aider les femmes à améliorer leur santé mentale (Chartier et al., 2012). Des programmes semblables pourraient donc apparaître tout aussi pertinents pour soutenir des personnes endeuillées par un suicide.

Si les formes de postvention active susmentionnées requièrent un lien maintenu en suivi individuel après un suicide, une autre approche pourrait, elle aussi, permettre de s'assurer que chacun reçoive l'aide nécessaire au moment où le suicide est découvert et pour préparer ainsi le terrain afin de faciliter l'accès à un soutien social. Former les premiers répondants tels que la police et les pompiers sur le traumatisme lié au suicide pourrait non seulement faciliter le soin immédiat des individus endeuillés, mais également ouvrir la voie à de futurs liens avec des services de soutien (Hawton, 2005). En plus de fournir aux personnes en deuil un accès immédiat à des services, comme par exemple une mise en relation avec un psychiatre, les "premiers répondants à un suicide» pourraient également fournir un dépliant ou une carte contenant des renseignements sur le soutien à la suite d'un suicide, soit un document qui se rapprocherait du livret conçu par l'organisme Speak Out (livret 32). Une approche similaire existe en 
Australie, où une unité mobile de réponse au suicide est disponible 24 heures sur 24. Elle offre ses services aux personnes endeuillées dès que le suicide est découvert, mais aussi un suivi des soins qui seront à leur disposition dans les douze mois qui suivent le décès (Hawton, 2005).

\section{Conclusion}

En conclusion, les politiques canadiennes sur le deuil à la suite d'un suicide sont déterminées par plusieurs facteurs institutionnels, comme l'influence des tribunaux, les compétences juridictionnelles respectives ou encore certains groupes qui ont des intérêts spécifiques. L'interaction de ces facteurs va alors conduire à des recommandations à partir desquelles les politiques peuvent être ensuite réorientées. Au Canada, de nos jours, le suicide n'est plus cet acte criminel stigmatisé d'autrefois. Il apparaît plutôt désormais comme un enjeu de santé publique nécessitant une prise en charge appropriée. Diverses approches (en amont, médianes et en aval) donnent ainsi des directions à suivre pour l'élaboration d'une politique de postvention du suicide. Davantage de recherches scientifiques devraient maintenant être menées dans le domaine de la prévention et de la postvention du suicide, et ce, en vue d'élargir nos connaissances non seulement sur le caractère adéquat ou non des soins proposés aux personnes endeuillées par un suicide mais aussi sur la pertinence des programmes en cours. Des avancées importantes aborderaient également le développement de la prévention et de la postvention du suicide au sein des Premières Nations. Surtout, elles devraient s'assurer que les politiques et programmes mis en place tiennent compte de la vision du monde, des perspectives et des pratiques de guérison de ces populations.

\section{Références}

Adams, E. F., Lee, A. J., Pritchard, C. W. et White, R. J. (2010). What stops us from healing the healers: A survey of help-seeking behaviour, stigmatisation and depression within the medical profession. International Journal of Social Psychiatry, 56(4), 359-370. doi: 0020764008099123

Agence de la santé publique du Canada. (2005). Initiatives et étapes clés à l'échelle nationale. Recherche sur le suicide au Canadd: Aperçu descriptif. Ottawa, Ontario. Repéré à https://www.canada.ca/fr/sante-publique/services/rapportspublications/recherche-suicide-canada-apercu-descriptif/initiativesnationale.html 
Agence de la santé publique du Canada. (2016). Coût des blessures au Canada. Repéré à https://www.canada.ca/fr/sante-publique/services/preventionblessures/cout-blessures-canada.html

Agerbo, E. (2005). Midlife suicide risk, partner's psychiatric illness, spouse and child bereavement by suicide or other modes of death: A gender specific study. J Epidemiol Community Health, 59(5), 407-412. doi: 10.1136/jech. 2004.024950

Ambrose, J. (1998). After suicide: reweaving the web. Dans A. A. Leenaars, S. Wenckstern, I. Sakinofsky, R. Dyck, M. Kral et R. C. Bland (dir.), Suicide in Canada (p. 385-405). Toronto, Ontario: University of Toronto Press.

Association canadienne pour la prévention du suicide. (2017a). CASP's purpose and function. Repéré à http://suicideprevention.ca/about-us/

Association canadienne pour la prévention du suicide. (2017b). Manitoba survivor support services. Repéré à http://suicideprevention.ca/manitobasurvivor-support-centres/

Association canadienne pour la prévention du suicide. (2017c). Suicide in Canada. Repéré à http://suicideprevention.ca/understanding/suicide-incanada/

Australian Government. (2008). Living is for everyone framework (2007). Barton, Australie: Commonwealth of Australia. Repéré à http://apo.org.au/ node/256.

Australian Government. (2009). Towards good practice: standards and guidelines for suicide bereavement support groups. Repéré à https://www.lifeline.org.au/ static/uploads/files/standards-and-guidelines-for-suicide-bereavementsupport-groups-wfcgrmzvzhaz.pdf

Beautrais, A. L. (2002). A case control study of suicide and attempted suicide in older adults. Suicide $\mathbb{F}$ life-threatening behavior, 32(1), 1-9.

Bolton, J. M., Au, W., Chateau, D., Walld, R., Leslie, W.D., Enns, J., ... Sareen, J. (2016). Bereavement after sibling death: A population-based longitudinal case-control study. World Psychiatry, 15(1), 59-66. doi : 10.1002/wps.20293

Bolton, J. M., Au, W., Leslie, W. D., Martens, P. J., Enns, M. W., Roos, L. L., ... Sareen, J. (2013). Parents bereaved by offspring suicide: A populationbased longitudinal case-control study. JAMA Psychiatry, 70(2), 158-167. doi: 10.1001/jamapsychiatry.2013.275

Bolton, J. M., Belik, S.-L., Enns, M. W., Cox, B. J. et Sareen, J. (2008). Exploring the correlates of suicide attempts among individuals with major depressive disorder: Findings from the national epidemiologic survey on alcohol and related conditions. J Clin Psychiatry, 69(7), 1139-1149. doi: ej07m03562 [pii]

Bombay, A., Matheson, K. et Anisman, H. (2011). The impact of stressors on second generation Indian residential school survivors. Transcult Psychiatry, 48(4), 367-391. doi: 10.1177/1363461511410240

Brent, D., Melhem, N., Donohoe, M. B. et Walker, M. (2009). The incidence and course of depression in bereaved youth 21 months after the loss of a parent to suicide, accident, or sudden natural death. Am J Psychiatry, 166(7), 786-794. doi : 10.1176/appi.ajp.2009.08081244 
Campbell, F. R., Cataldie, L., McIntosh, J. et Millet, K. (2004). An active postvention program. Crisis, 25(1), 30-32.

Cerel, J. et Campbell, F. R. (2008). Suicide survivors seeking mental health services: A preliminary examination of the role of an active postvention model. Suicide er life-threatening behavior, 38(1), 30-34. doi: 10.1521/ suli.2008.38.1.30

Cerel, J., McIntosh, J. L., Neimeyer, R. A., Maple, M. et Marshall, D. (2014). The continuum of "survivorship": Definitional issues in the aftermath of suicide. Suicide Life Threat Behav, 44(6), 591-600. doi : 10.1111/sltb.12093

Cerel, J., Padgett, J. H., Conwell, Y. et Reed, G. A. (2009). A call for research: The need to better understand the impact of support groups for suicide survivors. Suicide Life Threat Behav, 39(3), 269-281. doi: 10.1521/suli. 2009.39.3.269

Chartier, M., Finlayson, G., Prior, H., McGowan, K.-L., Chen, H., de Rocquigny, J., ... Gousseau, M. (2012). Health and bealthcare utilization of francophones in Manitoba/La santé et l'utilisation des services de santé des francophones du Manitoba. Winnipeg, Manitoba: Manitoba Centre for Health Policy.

Cheng, A. T., Chen, T. H., Chen, C. C. et Jenkins, R. (2000). Psychosocial and psychiatric risk factors for suicide. Case-control psychological autopsy study. Br J Psychiatry, 177, 360-365.

Commission de la santé mentale du Canada. (2011). Changer les mentalités. Repéré à https://www.mentalhealthcommission.ca/Francais/changer-les-mentalites

Commission de la santé mentale du Canada. (2012). Changer les orientations, changer des vies: Stratégie en matière de santé mentale pour le Canada. Repéré à https://www.mentalhealthcommission.ca/sites/default/files/MHStrategy_ Strategy_FRE.pdf

Connors, E. A., Rice, J. et Leenaars, A. A. (2017). Promoting a way of life to prevent premature death: Ojibway First Nation (Anishinaabe) healing practices. Dans K. Andriessen, K. Krysinska et O. Grad (dir.), Postvention in Action: The International Handbook of Suicide Bereavement Support (p. 255-265). Boston, MA: Hogrefe Publishing.

Constantino, R. E., Sekula, L. K. et Rubinstein, E. N. (2001). Group intervention for widowed survivors of suicide. Suicide $\mathcal{N}$ life-threatening behavior, 31(4), 428-441.

Conwell, Y., Duberstein, P. et Caine, E. (2002). Risk factors for suicide in later life. Biological Psychiatry, 52, 193-204.

Conwell, Y., Rotenberg, M. et Caine, E. (1990). Completed suicide at age 50 and over. Journal of the American Geriatrics Society, 38(6), 640-644.

Conwell, Y., Van Orden, K. et Caine, E. (2011). Suicide in older adults. Psychiatr Clin N Am, 34, 451-468.

Devries, K., Watts, C., Yoshihama, M., Kiss, L., Schraiber, L. B., Deyessa, N., ... Garcia-Moreno, C. (2011). Violence against women is strongly associated with suicide attempts: Evidence from the WHO multi-country study on women's health and domestic violence against women. Soc Sci Med, 73(1), 79-86. doi : 10.1016/j.socscimed.2011.05.006

Durkheim, E. (1952). Suicide: A study in sociology (traduit par J. A. Spaulding et G. Simpson). Londres, Royaume-Uni: Routledge et Kegan Paul Ltd. 
Edmunds, A. (1998). My story: Thoughts of a survivor. Dans A. A. Leenaars, S. Wenckstern, I. Sakinofsky, R. Dyck, M. Kral et R. C. Bland (dir.), Suicide in Canada (p. 369-375). Toronto, Ontario: University of Toronto Press.

Erlangsen, A., Runeson, B., Bolton, J. M., Wilcox, H. C., Forman, J. L., Krogh, J., ... Conwell, Y. (2017). Association between spousal suicide and mental, physical, and social health outcomes: A longitudinal and nationwide register-based study. JAMA Psychiatry, 74(5), 456-464. doi : 10.1001/jamapsychiatry. 2017.0226

Farberow, N. L., Gallagher-Thompson, D., Gilewski, M. et Thompson, L. (1992). Changes in grief and mental health of bereaved spouses of older suicides. J Gerontol, 47(6), P357-366.

Fung, Y. L. et Chan, Z. C. (2011). A systematic review of suicidal behaviour in old age: A gender perspective. Journal of Clinical Nursing, 20(15-16), 2109-2124.

Gouvernement du Canada. (1892). Criminal Code, 1892. Ottawa, Ontario. Repéré à https://ia902708.us.archive.org/27/items/criminalcodevic00canagoog/ criminalcodevic00canagoog.pdf

Gouvernement du Canada. (2009). Lois constitutionnelles de 1867 à 1982. Repéré à http://laws-lois.justice.gc.ca/fra/const/

Gouvernement du Canada. (2016). Travailler ensemble pour prévenir le suicide au Canada. Cadre fédéral de prévention du suicide. Repéré à https://www.canada. $\mathrm{ca} /$ content/dam/canada/public-health/migration/publications/healthyliving-vie-saine/framework-suicide-cadre-suicide/alt/framework-suicidecadre-suicide-fra.pdf

Gouvernement du Canada. (2017). Stratégie conjointe de prévention du suicide des Forces armées canadiennes et d'Anciens combattants Canada. Repéré à https:// www.canada.ca/content/dam/dnd-mdn/documents/reports/2017/strategieconjointe-prevention-suicide-fac-acc.pdf

Gravseth, H. M., Mehlum, L., Bjerkedal, T. et Kristensen, P. (2009). Suicide in young Norwegians in a life course perspective: Population based cohort study.J Epidemiol Community Health, 64(5), 407-412. doi : jech.2008.083485

Gureje, O., Oladeji, B., Hwang, I., Chiu, W. T., Kessler, R. C., Sampson, N. A., ... Nock, M. K. (2010). Parental psychopathology and the risk of suicidal behavior in their offspring: Results from the World Mental Health surveys. Molecular Psychiatry, 16(12), 1221-1233.

Hawton, K. (2005). Prevention and treatment of suicidal behavior: from science to practice. Oxford, Royaume-Uni : Oxford University Press.

Hawton, K. et van Heeringen, K. (2009). Suicide. Lancet, 373, 1372-1381.

Howlett, M., Ramesh, M. et Perl, A. (2009). Studying public policy (3 ${ }^{\mathrm{e}}$ éd.). Toronto, Ontario: Oxford University Press.

Indo European. (2011). The Swedish parliament has passed a law outlining tuition and application fees for students not from an EU/EEA country or Switzerland. Repéré à http://www.indoeuropean.in/images/112293.pdf

Instituts de recherche en santé du Canada. (2003). Report on the workshop on the suicide-related research in Canada-Montreal, February 7-8, 2003. Ottawa, Ontario. Repéré à http://www.phac-aspc.gc.ca/publicat/mh-sm/suicide-research/ pdf/suicide_research_e.pdf. 
Instituts de recherche en santé du Canada. (2006). Framework for action on mental illness and mental health: Recommendations to bealth and social policy leaders of canada for a national action plan on mental illness and mental health. Repéré à http://www.cmha.ca/data/1/rec_docs/601_CAMIMH English Lowres.pdf

Jordan, J. R. (2008). Bereavement after suicide. Psychiatric Annals, 38(10), 679685.

Jordan, J. R. (2015). Grief after suicide: The evolution of suicide postvention. New York, NY: Springer.

Jordan, J. R. et McIntosh, J. L. (2011a). Grief after suicide: Understanding the consequences and caring for the survivors. New York, NY: Routledge.

Jordan, J. R. et McIntosh, J. L. (2011b). Is suicide bereavement different? A framework for rethinking the question. Dans J. R. Jordan et J. McIntosh (dir.), Grief after suicide: understanding the consequences and caring for the survivors. New York, NY: Routledge.

Jordan, J. R. et McMenamy, J. (2004). Interventions for suicide survivors: A review of the literature. Suicide or life-threatening behavior, 34(4), 337-349. doi : 10.1521 /suli.34.4.337.53742

Jordan, J. R., Feigelman, W., McMenamy, J. et Mitchell, A. M. (2011). Research on the needs of survivors. Dans J. R. Jordan et J. L. McIntosh (dir.), Grief after suicide: understanding the consequences and caring for the survivors. New York, NY: Routledge.

Juurlink, D., Herrmann, N., Szalai, J., Kopp, A. et Redelmeier, D. A. (2004). Medical illness and the risk of suicide in the elderly. Archives of internal medicine, 164, 1179-1184.

Katz, L. Y., Au, W., Singal, D., Brownell, M., Roos, N., Martens, P. J., ... Sareen, J. (2011). Suicide and suicide attempts in children and adolescents in the child welfare system. CMAJ, 183(17), 1977-1981. doi : 10.1503/cmaj.110749

Katz, L. Y., Elias, B., O’Neil, J., Enns, M., Cox, B. J., Belik, S.-L. et Sareen, J. (2006). Aboriginal suicidal behaviour research: from risk factors to culturally-sensitive interventions. J Can Acad Child Adolesc Psychiatry, 15(4), 159167.

Kessing, L. V., Agerbo, E. et Mortensen, P. B. (2003). Does the impact of major stressful life events on the risk of developing depression change throughout life? Psychol Med, 33(7), 1177-1184.

Kessler, R. C., Borges, G. et Walters, E. E. (1999). Prevalence of and risk factors for lifetime suicide attempts in the National Comorbidity Survey. Archives of general psychiatry, 56(7), 617-626.

Kirkup, K. (2011). MPs call for national suicide prevention strategy, October 24. Cochrane Times Post. Repéré à http://www.cochranetimespost.ca/ 2011/10/04/mps-call-for-national-suicide-prevention-strategy-9

Kirmayer, L. J., Brass, G., Holton, T., Paul, T., Simpson, K. et Tait, C. L. (2007). Suicide Among Aboriginal People in Canada. Ottawa, Ontario: Aboriginal Healing Foundation. Repéré à http://www.ahf.ca/downloads/suicide.pdf

Kovac, S. H. et Range, L. M. (2000). Writing projects: Lessening undergraduates' unique suicidal bereavement. Suicide and Life-Threatening Behavior, $30(1), 50-60$. 
Kuramoto, J. S., Brent, D. A. et Wilcox, H. C. (2009). The impact of parental suicide on child and adolescent offspring. Suicide Life Threat Behav, 39(2), 137-151. doi : $10.1521 /$ suli.2009.39.2.137

Kuramoto, S. J., Stuart, E. A., Runeson, B. Lichtenstein, P., Långström, N. et Wilcox, H. C. (2010). Maternal or paternal suicide and offspring's psychiatric and suicide-attempt hospitalization risk. Pediatrics, 126(5), e1026-1032. doi : 10.1542 /peds.2010-0974

Lalonde, M. (1974). The new perspective on the health of Canadians. Ottawa, Ontario: Gouvernement du Canada. Repéré à http://www.phac-aspc.gc.ca/ ph-sp/pdf/perspect-eng.pdf.

Leenaars, A. A., Wenckstern, S., Sakinofsky, I., Dyck, R. J., Karl, M. J. et Bland, R. C. (1998). Suicide in Canada. Toronto, Ontario: University of Toronto Incorporated.

Mandelli, L., Carli, V., Roy, A., Serretti, A. et Sarchiapone, M. (2011). The influence of childhood trauma on the onset and repetition of suicidal behavior: An investigation in a high risk sample of male prisoners. Journal of Psychiatric Research, 45(6), 742-747.

Mann, J. J., Apter, A., Bertolote, J., Beautrais, A., Currier, D., Haas, A., ... Hendin, H. (2005a). Suicide prevention strategies: a systematic review. JAMA : Journal of the American Medical Association, 294(16), 2064-2074. doi : 10.1001/jama.294.16.2064

Mann, J. J., Bortinger, J., Oquendo, M. A., Currier, D., Li, S. et Brent, D. A. (2005b). Family history of suicidal behavior and mood disorders in probands with mood disorders. Am J Psychiatry, 162(9), 1672-1679.

Martens, P., Fransoo, R., The Need to Know Team, Burland, E. Prior, H., Burchill, C.,... Ouelette, C. (2008). What works? A first look at evaluating Manitoba's regional health programs and policies at the population level. Winnipeg, Manitoba: Manitoba Centre for Health Policy. Repéré à http://mchp-appserv.cpe. umanitoba.ca/reference/What \% 20Works.pdf

McDaid, C., Trowman, R., Golder, S., Hawton, K. et Sowden, A. (2008). Interventions for people bereaved through suicide: systematic review. The British Journal of Psychiatry, 193(6), 438-443. doi : 10.1192/bjp.bp.107.040824

McFarlane, J. M., Groff, J. Y., O’Brien, J. A. et Watson, K. (2003). Behaviors of children who are exposed and not exposed to intimate partner violence: an analysis of 330 black, white, and Hispanic children. Pediatrics, 112(3), e202-e207.

McKinlay, J. B. (1998). Paradigmatic obstacles to improving the health of populations-implications for health policy. Salud publica de Mexico, 40(4), 369-379.

McMenamy, J. M., Jordan, J. R. et Mitchell, A. M. (2008). What do suicide survivors tell us they need? Results of a pilot study. Suicide Life Threat Behav, 38(4), 375-389. doi: 10.1521/suli.2008.38.4.375

Miljan, L. (2008). Public policy in Canada (5 éd.). Don Mills, Ontario: Oxford University Press.

Nation Talk. (2010). NDP launches bill to create national suicide prevention strategy. Repéré à http://nationtalk.ca/story/ndp-launches-bill-to-createnational-suicide-prevention-strategy 
Nepon, J., Belik, S. L., Bolton, J. et Sareen, J. (2010). The relationship between anxiety disorders and suicide attempts: findings from the National Epidemiologic Survey on Alcohol and Related Conditions. Depress Anxiety, 27(9), 791-798. doi: 10.1002/da.20674

Nishimaki, S., Higuchi, R., Nakajima, S., Miyamae, T., Goto, H., Mori, M. et Yokota, S. (2009). Survey of psychological support for bereaved families in Japan. Pediatr Int, 51(5), 626-629. doi : 10.1111/j.1442-200X.2008.02802.x

Nock, M. K., Hwang, I., Sampson, N. A. et Kessler, R. C. (2010). Mental disorders, comorbidity and suicidal behavior: results from the National Comorbidity Survey Replication. Mol Psychiatry, 15(8), 868-876.

Nock, M. K., Hwang, I., Sampson, N., Kessler, R. C., Angermeyer, M., Beautrais, A., ... De Graaf, R. (2009). Cross-national analysis of the associations among mental disorders and suicidal behavior: findings from the WHO World Mental Health Surveys. PLoS Med, 6(8), e1000123. doi : 10.1371/ journal.pmed.1000123

Omerov, P., Steineck, G., Nyberg, T., Runeson, B. et Nyberg, U. (2013). Psychological morbidity among suicide-bereaved and non-bereaved parents: A nationwide population survey. BMJ open, 3(8), e003108

Organisation mondiale de la santé. (2002). Suicide Prevention in Europe: The WHO European Monitoring Survey on National Suicide Prevention Programmes and Strategies. Repéré à http://apps.who.int/iris/bitstream/handle/10665/ 107452/E77922.pdf; jsessionid =AA579CDA607AA49B00EF695E545CF $4 \mathrm{EF}$ ? sequence $=1$

Organisation mondiale de la santé. (2004). For which strategies of suicide prevention is there evidence of effectiveness? Repéré à http://www.euro.who.int/__ data/assets/pdf_file/0010/74692/E83583.pdf

Organisation mondiale de la santé. (2008). Preventing suicide: bow to start a survivor's group. Repéré à http://www.who.int/mental_health/prevention/ suicide/resource_survivors.pdf

Parlement du Canada. (1995). Le suicide chez les Autochtones: Le rapport de la Commission royale. Ottawa, Ontario. Repéré à http://publications.gc.ca/ collections/collection_2008/lop-bdp/mr/mr131-f.pdf

Penney, L. (2014). Self-determination and counselling to commit suicide: Beyond Rodriguez. Repéré à http://canliiconnects.org/en/commentaries/30674

Pfeffer, C. R., Jiang, H., Kakuma, T., Hwang, J. et Metsch, M. (2002). Group intervention for children bereaved by the suicide of a relative. Journal of the American Academy of Child and Adolescent Psychiatry, 41(5), 505-513. doi: 10.1097/00004583-200205000-00007

Pitman, A., Osborn, D., King, M. et Erlangsen, A. (2014). Effects of suicide bereavement on mental health and suicide risk. Lancet Psychiatry, 1(1), 86-94. doi : 10.1016/S2215-0366(14)70224-X

Public Initiative to Prevent Suicide and Self-Harm. (2007). The stigma of suicide: A history. Repéré à https://web.archive.org/web/20070317103747/http:// pipsproject.com/Understanding \% 20Suicide.html

Qin, P. et Mortensen, P. B. (2003). The impact of parental status on the risk of completed suicide. Archives of General Psychiatry, 60, 797-802. doi: 10.1001/archpsyc.60.8.797 
Qin, P., Agerbo, E. et Mortensen, P. B. (2003). Suicide risk in relation to socioeconomic, demographic, psychiatric, and familial factors: a national register-based study of all suicides in Denmark, 1981-1997. The American Journal of Psychiatry, 160(4), 765-772.

Rose, G. (1985). Sick individuals and sick populations. International Journal of Epidemiology, 14(1), 32-38.

Rosenfeld, L. (1998). I can't hear the music. Dans A. A. Leenaars, S. Wenckstern, I. Sakinofsky, R. Dyck, M. Kral et R. C. Bland (dir.), Suicide in Canada (p. 376-384). Toronto, Ontario: University of Toronto Press.

Russell, S. et Joyner, K. (2001). Adolescent sexual orientation and suicide risk: Evidence from a national study. American Journal of Public Health., 91(8), 1276-1281.

Santé Canada. (1987). Suicide in Canada. Ottawa, Ontario: Santé Canada.

Santé Canada. (2003). Acting on what we know: preventing youth suicide in First Nations: the report of the advisory group on suicide prevention. Repéré à http:// publications.gc.ca/site/eng/239048/publication.html

Sapsford, L. (1998). Women as survivors of suicide: an experience of integration. Dans A. A. Leenaars, S. Wenckstern, I. Sakinofsky, R. Dyck, M. Kral et R. Bland (dir.), Suicide in Canada (p. 406-422). Toronto, Ontario: University of Toronto Incorporated.

Sareen, J., Jagdeo, A., Cox, B. J., Clara, I., ten Have, M., Belik, S.-L., ... Stein, M. B. (2007). Perceived barriers to mental health service utilization in the United States, Ontario, and the Netherlands. Psychiatric services, 58(3), 357364. doi : 10.1176/appi.ps.58.3.357

Schaal, S., Elbert, T. et Neuner, F. (2009). Prolonged grief disorder and depression in widows due to the Rwandan genocide. Omega, 59(3), 203-219.

Sénat du Canada. (2005). Délibérations du comité sénatorial permanent des Affaires sociales, des sciences et de la technologie. Charlottetown, Île-du-Prince-Édouard. Repéré à https://sencanada.ca/fr/Content/Sen/committee/381/soci/22evc-f

Sénat du Canada. (2006). Proceedings of the Standing Senate Committee on Social Affairs, Science and Technology. Ottawa, Ontario. Repéré à http://www.parl. gc.ca/Content/SEN/Committee/391/soci/rep/rep02may06-e.htm

Shneidman, E. S. (1981). The psychological autopsy. Suicide Life Threat Behav, 11, 325-340.

Sommer-Rotenberg, D. (1998). Suicide and language. CMAJ, 159(3), 239-240.

Sorensen, H. J., Mortensen, E. L., Wang, A. G., Juel, K., Silverton, L. et Mednick, S. A. (2009). Suicide and mental illness in parents and risk of suicide in offspring: a birth cohort study. Soc Psychiatry Psychiatr Epidemiol, 44(9), 748-751. doi : 10.1007/s00127-009-0495-5

Spiwak, R., Elias, B., Bolton, J. M., Martens, P. J. et Sareen J. (2012). Suicide policy in Canada: lessons from history. CanJ Public Health, 103(5), e338-341.

Spiwak, R., Pagura, J., Bolton, J., Ealias, B., Beesdo-Baum, K., Lieb, R. et Sareen, J. (2011). Childhood exposure to caregiver suicidal behavior and risk for adult suicide attempts: findings from a national survey. Archives of Suicide Research, 15(4), 313-326. 
Sveen, C. A. et Walby, F. A. (2008). Suicide survivors' mental health and grief reactions: A systematic review of controlled studies. Suicide Life Threat Behav, 38(1), 13-29. doi: 10.1521/suli.2008.38.1.13

Swampy Cree Suicide Prevention Team. (2008). Swampy Cree Suicide Prevention Team. A University-First Nations Community Partnership. Repéré à http://www.suicideresearch.ca

Szumilas, M. et Kutcher, S. (2011). Post-suicide intervention programs: A systematic review. Canadian Public Health Association, 102(1), 18-29.

Tanney, B. (1995). Suicide prevention in Canada: A national perspective highlighting progress and problems. Suicide Life Threat Behav, 25(1), 105-122.

Tidemalm, D., Runeson, B., Waern, M., Frisell, T., Carlström, E., Lichtenstein, P. et Langstrom, N. (2011). Familial clustering of suicide risk : a total population study of 11.4 million individuals. Psychol Med, 41(12), 2527-2534. doi : $10.1017 /$ S0033291711000833

Van Orden, K. et Conwell, Y. (2011). Suicides in late life. Curr Psychiatry Rep, 13(3), 234-241. doi : 10.1007/s11920-011-0193-3

von Borczyskowski, A., Lindbland, F., Vinnerlijung, B., Reintjes, R. et Hjern, A. (2011). Familial factors and suicide: an adoption study in a swedish national cohort. Psychological Medicine, 41(4), 749-758.

Waern, M., Rubenowitz, E. et Runeson, B. (2002). Burden of illness and suicide in elderly people: case control study. British Medical Journal, 324, 1-4.

Yin Bun, C., Law, C. K., Chan, B., Liu, K. Y. et Yip, P. S. (2006). Suicidal ideation and suicidal attempts in a population based study of Chinese people: Risk attributable to hopelessness, depression and social factors. Journal of Affective Disorders, 90(2), 193-199.

Youth Suicide Advisory Committee. (1998). Recommended policy and programs for preventing suicide and suicidal behavior among Aboriginal Youth in Western Australia. Repéré à http://www.healthinfonet.ecu.edu.au/key-resources/ bibliography? page $=7 \& \mathrm{q}=\& \mathrm{q} \_$exact $=\& \mathrm{q} \_$author $=\&$ as_values_tagged _keyword $=\&$ sorter $=$ author-DESC \&year_start $=1840$ \&year_end $=$ 2017\&lid $=21749$.

\section{A new public health issue in Canada: Helping people bereaved by suicide}

ABSTRAC - Worldwide, between 48 and 500 million individuals are affected by suicide every year. In Canada alone, 4,000 individuals die yearly by suicide, meaning that a substantial portion of the population must cope with the traumatic and complex aftermath of suicide. This paper discusses the emergence of suicide bereavement as a public health issue following the decriminalization of attempted suicide in 1972. The role of key structures, including the courts, the constitution, and special interest groups, that have shaped suicide and suicide bereavement policy in Canada are examined. Health outcomes associated with suicide bereavement are reviewed, as well as the need for intervention. Emerging areas of suicide policy where guidance is needed are also discussed, as are the need for upstream, midstream, and downstream approaches to suicide bereavement intervention.

KEYWORDS - Suicide, policy, prevention, Canada. 


\section{Una nueva apuesta de salud publica en Canadá. El acompañamiento de las personas afligidas después de un suicidio}

RESUMEN - A nivel mundial, entre 48 y 500 millones de individuos sufren el impacto del duelo a causa de un suicidio anualmente. Solamente en Canadá, 4000 individuos mueren anualmente por causa del suicidio, lo cual significa que una proporción substancial de la población debe afrontar el traumatismo y la complejidad de la naturaleza del suicidio. Este artículo trata de la emergencia del duelo frente al suicidio como un asunto de salud pública después de que fuese votada la descriminalización de la tentativa de suicidio en 1972. Examinamos aquí el rol de estructuras claves, incluyendo las cortes, la constitución y los grupos de interés especiales, que le ha modelado el suicidio y la política del duelo frente al suicidio en Canadá. Las consecuencias para la salud, asociadas al duelo frente al suicidio son revisadas, asi como la necesidad de intervención. Áreas emergentes de la política del suicidio en las que una guía es requerida son también discutidas, agregándose a la necesidad de aproximaciones a corto, mediano y largo plazo, en lo que se refiere a la intervención frente al duelo.

PALABRAS CLAVE • Suicidio, política, prevención, Canadá. 